\title{
Association between MFN2 gene polymorphisms and the risk and prognosis of acute liver failure: a case-control study in a Chinese population
}

\author{
Y.-L. Wei, Q. Tian, X.-X. Zhao, G.-Z. Qiu and Y. Xu \\ Department of Infectious Diseases, Linyi People's Hospital, Linyi, China
}

\begin{abstract}
This study aimed to determine the role of mitofusin 2 (MFN2) gene polymorphisms in the risk and prognosis of acute liver failure (ALF). A total of 298 blood samples were collected from 138 ALF patients (case group) and 160 healthy participants (control group). Coagulation function, glutamic pyruvic transaminase (GPT), glutamic oxaloacetic transaminase (GOT), total bilirubin (TB), blood ammonia and lactic acid (LA) were measured. The predictive evaluation of MFN2 gene polymorphisms in the risk and prognosis of ALF patients was estimated using Kaplan-Meier survival analysis, haplotype analysis, binary logistic regression analysis and Cox regression analysis. Higher levels of GPT, GOT, TB, blood ammonia and LA were observed in ALF patients with the GG genotype of rs873457 or the TT genotype of rs4846085 than in those with the CC genotype of these two SNPs. The GTACAGC and GTGTGGC haplotypes were a protective factor and a risk factor for ALF, respectively. Blood ammonia and LA levels were independent risk factors and the CC genotype of rs 873457 and the CC genotype of rs 4846085 were protective factors for ALF. ALF patients with the GG genotype of rs873457 or the TT genotype of rs4846085 had a lower survival rate than those with other genotypes of these two SNPs. The rs 4846085 and rs873457 polymorphisms were both independent factors affecting the prognosis of ALF patients. MFN2 gene polymorphisms (rs873457, rs2336384, rs1474868, rs4846085 and rs2236055) may be associated with ALF and the rs873457 and rs4846085 polymorphisms are correlated with the risk and prognosis of ALF.
\end{abstract}

Key words: Acute liver failure; MFN2; Gene polymorphism; Risk; Prognosis; Chinese population

\section{Introduction}

Acute liver failure (ALF) is defined as severe hepatocyte necrosis and liver injury followed by hepatic encephalopathy within 8 weeks without any pre-existing liver disease (1). ALF often occurs in healthy adults aged approximately 30 , and its incidence is approximately $10 /$ $1,000,000$ per year in developed countries (2). Patients with ALF usually show elevated bilirubin level and transaminase activity and reduced levels of blood coagulation factors, including factor $\mathrm{V}$ Leiden and prothrombin. Regarding ALF pathogenesis, viral infection, drug metabolism, cancer, adipose infiltration and irradiation injuries can contribute to abnormal liver cell metabolism, which ultimately results in liver failure (3). A recent report has demonstrated that endogenous factors, including mitofu$\sin 1$ (MFN1) and mitofusin 2 (MFN2), are correlated with the occurrence of ALF (4).

The MFN2 gene encodes a mitochondrial membrane protein that takes part in mitochondrial fusion (5). The MFN2 protein is located on the outer membrane of the mitochondria and has GTPase activity. The mitochondria are the main organelles in charge of energy metabolism and reactive oxygen species generation, and mitochondrial damage may contribute to liver diseases (6). As the MFN2 gene primarily acts on the regulation of mitochondrial fusion, division, distribution, shape maintenance, and metabolic activity, aberrant MFN2 gene expression can lead to an increase in oxidative stress, which causes liver cell damage (7). The MFN2 gene has been shown to be correlated with several liver diseases, including fatty liver and liver failure (8). Previous studies have shown that single-nucleotide polymorphisms (SNPs) in the MFN2 gene may regulate the function or expression of MFN2 and that over-expression of MFN2 reduces liver ischemiareperfusion injury $(9,10)$. Although studies have focused on the relationship between MFN2 gene polymorphisms and many diseases, such as essential hypertension (11) and axonal Charcot-Marie-Tooth disease (CMT2) (12), its correlation with ALF remains unknown. Therefore, the relationship between MFN2 gene polymorphisms and ALF has been extensively explored in this study.

Correspondence: Q. Tian: <drtianq@163.com> 


\section{Material and Methods}

\section{Subjects}

Blood samples ( $n=298)$ were collected from 138 patients with ALF (case group) admitted to Linyi People's Hospital from January 2010 to January 2015 and from 160 healthy participants (control group). Among the patients, there were 112 males and 26 females ranging in age from 20 to 50 , and mean age of $37.33 \pm 4.94$ years. In addition, in terms of pathogenesis, there were 105 suffering from viral hepatitis [8 $(7.6 \%)$ were infected with hepatitis $A$ virus, $84(80.0 \%)$ with hepatitis B virus (HBV), 7 (6.7\%) with hepatitis $C$ virus, 5 (4.8\%) with hepatitis $E$ virus (HEV) and $1(1 \%)$ with HBV and HEV]; 22 (21\%) suffered from drug intoxication; and 11 (10.5\%) had pregnancy-related ALF. All 138 ALF patients received diverse conservative treatments based on the different causes; these treatments included drug therapy such as the use of nucleoside analogues (e.g., lamivudine, telbivudine and entecavir), hormone therapy such as the use of glucocorticoids, nutritional support, therapies that promote hepatocyte regeneration, and oral probiotics or lactulose oral solution to protect the intestinal tract. In addition, 58 patients were treated with plasma-exchange therapy in an artificial liver support system. Diagnostic criteria $(13,14)$ were as follows: acute onset hepatic encephalopathy of grade II to IV (I-IV classification) occurring within 2 weeks and accompanied by the following symptoms: 1) extreme fatigue and severe digestive tract symptoms, including anorexia, lethargy, abdominal distension, nausea and emesis; 2) gradual onset of jaundice; 3) coagulopathy [increased bleeding tendency and prothrombin time $<40 \mathrm{~s}$ (international normalized ratio (INR) $\geqslant 1.5$ ), but other causes that led to uncontrolled gingival bleeding, gastrointestinal bleeding or bleeding hemorrhoids were excluded]; 4) overt conjugated hyperbilirubinemia, elevated aminotransferases (>10,000 IU/U); 5) progressive liver shrinking, and 6) right upper quadrant tenderness. Exclusion criteria included 1) coinfection with other hepatotropic viruses or non-hepatotropic viruses; 2) alcoholic, autoimmune or drug-induced hepatitis; 3 ) ascites detected by B ultrasound; 4) liver cirrhosis detected by imaging examinations; 5) infection with bacteria or fungi, and 6) history of high blood pressure, heart disease, diabetes, kidney disease or other chronic diseases. A total of 160 healthy individuals were selected as a control group, including 129 males and 31 females ranging in age from 19-55 years, and mean age of $36.82 \pm 5.43$ years. The basic clinical information of all subjects was obtained, and their coagulation function (using INR), glutamic pyruvic transaminase (GPT), glutamic oxaloacetic transaminase (GOT), total bilirubin (TB), blood ammonia and lactic acid (LA) levels were determined. The study was approved by the Ethics Committee of Linyi People's Hospital and all participants signed an informed consent form.

\section{DNA extraction and SNP selection}

MFN2 SNPs in the SNP NCBI Database (http://www. ncbi.nlm.nih.gov/sites/entrez?db=Snp) with a frequency over $5 \%$ were selected, and tagging SNPs were searched in the HapMap website (http://www.hapmap.org/index.html. en) to determine the SNPs used in the present study. DNA was extracted from blood samples $(5 \mathrm{~mL})$ using a Purgene kit (Gentra Systems, USA) according to the manufacturer's protocol and was stored at $-20^{\circ} \mathrm{C}$. The MFN2 gene SNPs were analyzed with a SNP genotyping kit purchased from Applied Biosystems (Applied Biosystems Inc., USA). A pair of primers and a TaqMan probe were included in the kit. Seven SNPs were chosen according to previous references: rs2236055, rs4240897, rs1474868, rs2336384, rs873458, rs873457 and rs484608, corresponding to C_15953632_10 (rs2236055), C_26207636_10 (rs4240897), C_1267235_20 (rs1474868), C_11461995_10 (rs2336384), C_8861262_10 (rs873458), C_11461996_20 (rs873457), C_1267226_10 (rs4846085). A 7300 Real-Time PCR System was used for PCR amplification, and the program was set as follows: $95^{\circ} \mathrm{C}$ for $10 \mathrm{~min}$, followed by 40 cycles of $95^{\circ} \mathrm{C}$ for $15 \mathrm{~s}$, and $60^{\circ} \mathrm{C}$ for $60 \mathrm{~s}(9,11)$.

\section{Follow-up}

A 24-week follow-up was conducted on all patients after hospital discharge, and the end of follow-up was June 2016. Telephone calls, outpatient services and medical records were utilized to conduct the follow-up, and no patient was lost. Survival time was calculated in weeks, and all the patients, including those who died during hospitalization, were taken into consideration.

\section{Statistical analysis}

All statistical analyses were performed using SPSS 20.0 (SPSS Inc., USA). Data are reported as means \pm SD, and comparisons between two groups were performed using Student's $t$-test. The Hardy-Weinberg equilibrium test was used for SNP analysis. Binary logistic regression analysis was used to estimate the associations between SNPs and ALF. Haplotype correlation analysis was performed using SHEsis software (http://analysis.bio-x.cn/ myAnalysis.php). The survival analysis was conducted using the Kaplan-Meier survival curve method and confirmed by log-rank tests. Patient prognosis was analyzed by Cox proportional hazards survival regression. $\mathrm{P}<0.05$ was considered statistically significant.

\section{Results}

\section{Comparison of baseline characteristics between ALF patients and healthy participants}

As demonstrated in Table 1, no significant difference in gender, age or BMI was found between ALF patients and healthy participants. The coagulation function, GPT, GOT, TB, blood ammonia and LA levels in ALF patients were 
Table 1. Comparison of baseline characteristics between acute liver failure patients and healthy participants.

\begin{tabular}{|c|c|c|c|c|c|}
\hline Characteristic & Normal reference & Control group $(n=160)$ & Case group $(n=138)$ & $\chi^{2} / \mathrm{t}$ & $\mathrm{P}$ \\
\hline Gender (male/female) & & $129 / 31$ & $112 / 26$ & 0.117 & 0.907 \\
\hline Age (years) & & $36.82 \pm 5.32$ & $37.33 \pm 4.94$ & 0.853 & 0.394 \\
\hline BMI $\left(\mathrm{kg} / \mathrm{m}^{2}\right)$ & $18.5-24.99$ & $24.37 \pm 3.74$ & $25.06 \pm 3.59$ & 1.618 & 0.107 \\
\hline GPT (U/L) & $0-40$ & $33.87 \pm 5.22$ & $119.86 \pm 19.83$ & 52.78 & $<0.001$ \\
\hline GOT (U/L) & $8-40$ & $28.69 \pm 2.48$ & $151.07 \pm 18.81$ & 81.50 & $<0.001$ \\
\hline $\mathrm{TB}(\mu \mathrm{mol} / \mathrm{L})$ & $3.4-17.1$ & $12.59 \pm 2.34$ & $330.34 \pm 35.55$ & 112.80 & $<0.001$ \\
\hline Blood ammonia $(\mu \mathrm{mol} / \mathrm{L})$ & $20-60$ & $55.06 \pm 8.07$ & $87.40 \pm 9.81$ & 31.22 & $<0.001$ \\
\hline $\mathrm{LA}(\mathrm{mmol} / \mathrm{L})$ & $0.5-1.7$ & $1.15 \pm 0.46$ & $4.96 \pm 2.39$ & 19.75 & $<0.001$ \\
\hline
\end{tabular}

Data are reported as mean \pm SD. BMI: body mass index; GPT: glutamic pyruvic transaminase; GOT: glutamic oxaloacetic transaminase; TB: total bilirubin; LA: lactic acid. The chi-square test or the $t$-test was used for statistical analysis.

significantly higher than those in the healthy participants (all $\mathrm{P}<0.05$ ).

\section{SNP selection of the MFN2 gene}

MFN2 SNPs were obtained from the NCBI database. The genotype frequencies and theoretical frequencies of the seven SNPs were in accordance with Hardy-Weinberg equilibrium (both $\mathrm{P}>0.05$ ). The MFN2 SNP selection showed that at rs873457, rs2336384, rs1474868, rs4846085 and rs2236055, the genotype and allele distributions were significantly different between case and the control groups (all $\mathrm{P}<0.05$; Table 2), while the rs873458 and rs4240897 SNPs showed no significant difference (both $P>0.05$ ). At rs873457, the CC genotype and the $C$ allele were more frequent in the control group than in the case group, and the $\mathrm{C}$ allele was associated with reduced ALF risk compared with the $\mathrm{G}$ allele $(\mathrm{P}<0.05$, OR=0.601, 95\% $\mathrm{Cl}=0.434$ 0.833). At rs2336384, the frequencies of the TT genotype and $T$ allele were significantly higher in the control group compared with the case group, and T allele was also associated with decreased ALF risk compared with the $G$ allele $(\mathrm{P}<0.05, \mathrm{OR}=0.683,95 \% \mathrm{Cl}=0.494-0.943)$. For $\mathrm{rs} 1474868$, the $G G$ genotype and $G$ allele occurred more frequently in the control group than in the case group, and the $G$ allele was associated with lower ALF risk compared with the A allele $(\mathrm{P}<0.05, \mathrm{OR}=0.659,95 \% \mathrm{Cl}=0.478-0.912)$. At rs4846085, the CC genotype and C allele were more frequently found in the control group than in the case group, indicating that the $C$ allele may be a protective factor for ALF compared with the $T$ allele $(P<0.05, O R=0.660$, $95 \% \mathrm{Cl}=0.478-0.913)$. At rs2236055, the GG genotype and $\mathrm{G}$ allele occurred more frequently in the control group than in the case group, and the $G$ allele may lower the risk of ALF compared with the A allele $(P<0.05, O R=0.663$, $95 \% \mathrm{Cl}=0.480-0.916)$.

\section{Comparison of liver function in ALF patients with different genotypes of MFN2 polymorphisms}

We further analyzed the correlations between different genotypes of these 5 SNPS and liver function indexes.
As shown in Table 3, patients with the GG genotype of rs873457 or the TT genotype of rs4846085 had higher coagulation function, GPT, GOT, TB, blood ammonia and LA levels compared with the other genotypes (all $\mathrm{P}<$ 0.05 ). There were no significant correlations between liver function indexes and the different genotypes of the other 3 SNPs (all $P>0.05$ ). All patients had an INR $\geqslant 1.5$.

\section{Correlation analysis between haplotypes of MFN2 gene polymorphisms and ALF risk}

The correlation between haplotypes of MFN2 SNPs and ALF was analyzed by SHEsis software (Table 4). Haplotypes with frequencies $<3 \%$ in both groups were not considered. The results showed that among the seven common haplotypes, GTACAGC at rs 2236055 was a protective factor for ALF (OR=0.080, 95\% Cl=0.014-0.443, $\mathrm{P}<0.05$ ), while GTGTGGC at $\mathrm{rs} 873458$ was a risk factor for ALF (OR=5.644, 95\%Cl=1.846-17.258, $\mathrm{P}<0.05)$. No other haplotypes showed correlations with ALF $(P>0.05)$.

\section{Binary logistic regression analysis of ALF risk factors}

The correlations between MFN2 gene polymorphisms and liver function indexes with ALF were analyzed by binary logistic regression (Table 5 ). The results indicated that blood ammonia $(\mathrm{P}<0.05, \mathrm{OR}=1.636,95 \% \mathrm{Cl}=1.364-$ 1.962) and $L A$ levels $(P<0.05$, OR $=10.591,95 \% \mathrm{Cl}=5.364$ 20.912) were independent risk factors for ALF, while the CC genotype of rs873457 $(P<0.05, O R=0.375,95 \%$ $\mathrm{Cl}=0.221-0.634)$ and the $\mathrm{CC}$ genotype of rs 4846085 were protective factors for ALF. Other functional parameters, including GPT, GOT and TB ( $\mu \mathrm{mol} / \mathrm{L})$, were not associated with ALF (P>0.05).

\section{Relationship between MFN2 gene polymorphisms and short-term prognosis of ALF patients}

One hundred and thirty-eight patients were followedup for 24 weeks (100\% follow-up rate). During this period, 83 died, and 55 survived, giving a death rate of $60.15 \%$. The relationship between SNPs of the MFN2 gene and the risk of ALF was analyzed. The results showed a 
Table 2. Distribution of genotype and allele frequencies of MFN2 gene polymorphisms in acute liver failure patients and healthy participants.

\begin{tabular}{|c|c|c|c|c|c|c|}
\hline SNP & SNP/Genotype/Allele & Control group $(n=160)$ & Case group $(n=138)$ & $\mathrm{P}$ & OR & $95 \% \mathrm{Cl}$ \\
\hline \multicolumn{7}{|l|}{ rs873457 } \\
\hline \multirow[t]{3}{*}{ Genotype } & GG & $32(20.0 \%)$ & $35(25.4 \%)$ & & 1 (Ref.) & \\
\hline & GC & $65(40.6 \%)$ & $76(55.1 \%)$ & 0.822 & 1.069 & $0.596-1.195$ \\
\hline & $\mathrm{CC}$ & $63(39.4 \%)$ & 27 (19.5\%) & 0.005 & 0.392 & $0.203-0.757$ \\
\hline \multirow[t]{2}{*}{ Allele } & $\mathrm{G}$ & $129(40.3 \%)$ & $146(52.9 \%)$ & & 1 (Ref.) & \\
\hline & C & $191(59.7 \%)$ & $130(47.1 \%)$ & 0.002 & 0.601 & $0.434-0.833$ \\
\hline \multicolumn{7}{|l|}{ rs2336384 } \\
\hline \multirow[t]{3}{*}{ Genotype } & GG & $34(21.3 \%)$ & $36(26.1 \%)$ & & 1 (Ref.) & \\
\hline & GT & $72(45.0 \%)$ & $75(54.3 \%)$ & 0.955 & 0.984 & $0.557-1.739$ \\
\hline & TT & $54(33.7 \%)$ & $27(19.6 \%)$ & 0.019 & 0.458 & $0.237-0.888$ \\
\hline \multirow[t]{2}{*}{ Allele } & G & $140(43.7 \%)$ & $147(53.3 \%)$ & & 1 (Ref.) & \\
\hline & $\mathrm{T}$ & $180(56.3 \%)$ & $129(46.7 \%)$ & 0.021 & 0.683 & $0.494-0.943$ \\
\hline \multicolumn{7}{|l|}{ rs1474868 } \\
\hline \multirow[t]{3}{*}{ Genotype } & $A A$ & $29(18.1 \%)$ & $35(25.4 \%)$ & & 1 (Ref.) & \\
\hline & AG & $77(48.1 \%)$ & $75(54.3 \%)$ & 0.473 & 0.807 & $0.449-1.450$ \\
\hline & GG & $54(33.8 \%)$ & $28(20.3 \%)$ & 0.013 & 0.43 & $0.220-0.841$ \\
\hline \multirow[t]{2}{*}{ Allele } & A & $135(42.2 \%)$ & $145(52.5 \%)$ & & 1 (Ref.) & \\
\hline & $\mathrm{G}$ & $185(57.8 \%)$ & 131 (48.9\%) & 0.012 & 0.659 & $0.478-0.912$ \\
\hline \multicolumn{7}{|l|}{ rs4846085 } \\
\hline \multirow[t]{3}{*}{ Genotype } & TT & $31(19.4 \%)$ & $38(27.5 \%)$ & & 1 (Ref.) & \\
\hline & TC & $80(50.0 \%)$ & 75 (54.3\%) & 0.356 & 0.765 & $0.433-1.352$ \\
\hline & $\mathrm{CC}$ & $49(30.6 \%)$ & $25(18.2 \%)$ & 0.01 & 0.416 & $0.212-0.819$ \\
\hline \multirow[t]{2}{*}{ Allele } & $\mathrm{T}$ & $142(44.4 \%)$ & $151(54.7 \%)$ & & 1 (Ref.) & \\
\hline & C & 178 (55.6\%) & $125(45.3 \%)$ & 0.012 & 0.66 & $0.478-0.913$ \\
\hline \multicolumn{7}{|l|}{ rs4240897 } \\
\hline \multirow[t]{3}{*}{ Genotype } & AA & $29(18.1 \%)$ & $30(21.7 \%)$ & & 1 (Ref.) & \\
\hline & $A G$ & 85 (53.1\%) & $81(58.7 \%)$ & 0.786 & 0.921 & $0.508-1.669$ \\
\hline & GG & $46(28.8 \%)$ & 27 (19.6\%) & 0.11 & 0.567 & $0.283-1.140$ \\
\hline \multirow[t]{2}{*}{ Allele } & A & $143(44.7 \%)$ & $141(51.1 \%)$ & & 1 (Ref.) & \\
\hline & G & $177(55.3 \%)$ & $135(48.9 \%)$ & 0.119 & 0.774 & $0.560-1.068$ \\
\hline \multicolumn{7}{|l|}{ rs2236055 } \\
\hline \multirow[t]{3}{*}{ Genotype } & AA & $27(16.9 \%)$ & $35(25.4 \%)$ & & 1 (Ref.) & \\
\hline & $A G$ & $78(48.8 \%)$ & $74(53.6 \%)$ & 0.303 & 0.732 & $0.404-1.326$ \\
\hline & GG & 55 (34.4\%) & $29(21.0 \%)$ & 0.008 & 0.407 & $0.207-0.798$ \\
\hline \multirow[t]{2}{*}{ Allele } & A & $136(42.5 \%)$ & $144(52.2 \%)$ & & 1 (Ref.) & \\
\hline & G & $188(58.8 \%)$ & $132(47.8 \%)$ & 0.013 & 0.663 & $0.480-0.916$ \\
\hline \multicolumn{7}{|l|}{ rs873458 } \\
\hline \multirow[t]{3}{*}{ Genotype } & TT & $20(12.5 \%)$ & $24(17.4 \%)$ & & 1 (Ref.) & \\
\hline & TC & $79(49.4 \%)$ & $67(48.6 \%)$ & 0.314 & 0.707 & $0.359-1.391$ \\
\hline & $\mathrm{CC}$ & $61(38.1 \%)$ & 47 (34.0\%) & 0.217 & 0.642 & $0.317-1.300$ \\
\hline \multirow[t]{2}{*}{ Allele } & $\mathrm{T}$ & $119(37.2 \%)$ & $115(41.5 \%)$ & & 1 (Ref.) & \\
\hline & C & $201(62.8 \%)$ & $161(58.5 \%)$ & 0.264 & 0.829 & $0.596-1.153$ \\
\hline
\end{tabular}

SNP: single nucleotide polymorphism; OR: Odds ratio; Cl: confidence interval. Statistical analysis was carried out with the chi-square test.

significantly lower survival rate in ALF patients with the GG genotype of rs873457 than those with the GC + CC genotypes (Figure 1A; $P<0.05$ ). Patients with the TT genotype of rs4846085 had a remarkably lower survival rate than those with the $\mathrm{TC}+\mathrm{CC}$ genotypes (Figure 1B; $\mathrm{P}<0.05)$.

\section{Cox proportional hazards survival regression of short-term prognosis of ALF patients}

As shown in Table 6, patients' clinical factors and MFN2 polymorphisms were introduced into a Cox regression model. The results showed that GPT, GOT, TB, blood ammonia and LA were not associated with the prognosis 
Table 3. Comparison of liver functions in acute liver failure patients with different genotypes of MFN2 gene polymorphisms.

\begin{tabular}{|c|c|c|c|c|c|}
\hline SNP/Genotype & GPT (U/L) & GOT (U/L) & TB $(\mu \mathrm{mol} / \mathrm{L})$ & Blood ammonia $(\mu \mathrm{mol} / \mathrm{L})$ & $\mathrm{LA}(\mathrm{mmol} / \mathrm{L})$ \\
\hline \multicolumn{6}{|l|}{ rs873457 } \\
\hline GG & $124.45 \pm 18.31$ & $156.75 \pm 20.24$ & $348.94 \pm 37.27$ & $89.52 \pm 7.73$ & $5.5 \pm 2.69$ \\
\hline GC & $124.69 \pm 17.21$ & $154.82 \pm 16.76$ & $334.66 \pm 26.59$ & $90.56 \pm 8.25$ & $5.28 \pm 2.27$ \\
\hline $\mathrm{CC}$ & $100.33 \pm 16.94^{*}$ & $133.12 \pm 9.95^{*}$ & $294.09 \pm 30.33^{*}$ & $75.74 \pm 7.52^{*}$ & $3.38 \pm 1.53^{*}$ \\
\hline \multicolumn{6}{|l|}{ rs2336384 } \\
\hline GG & $123.32 \pm 14.34$ & $147.61 \pm 16.89$ & $330.58 \pm 30.81$ & $87.38 \pm 10.69$ & $4.41 \pm 2.41$ \\
\hline GT & $117.2 \pm 21.37$ & $152.08 \pm 19.91$ & $327.53 \pm 34.39$ & $86.87 \pm 9.92$ & $5.01 \pm 2.28$ \\
\hline TT & $122.65 \pm 21.20$ & $152.87 \pm 18.12$ & $337.83 \pm 44.00$ & $88.89 \pm 8.38$ & $5.57 \pm 2.56$ \\
\hline \multicolumn{6}{|l|}{ rs1474868 } \\
\hline AA & $116.74 \pm 15.15$ & $150.07 \pm 21.13$ & $330.13 \pm 33.92$ & $88.11 \pm 10.42$ & $4.71 \pm 2.48$ \\
\hline$A G$ & $122.11 \pm 20.16$ & $151.60 \pm 17.12$ & $329.81 \pm 38.47$ & $88.07 \pm 9.10$ & $5.13 \pm 2.43$ \\
\hline GG & $117.75 \pm 23.68$ & $150.88 \pm 20.54$ & $332.04 \pm 30.10$ & $84.71 \pm 10.75$ & $4.83 \pm 2.19$ \\
\hline \multicolumn{6}{|l|}{ rs4846085 } \\
\hline TT & $123.76 \pm 15.41$ & $152.90 \pm 17.84$ & $335.51 \pm 28.68$ & $91.86 \pm 8.38$ & $4.93 \pm 2.31$ \\
\hline $\mathrm{TC}$ & $124.95 \pm 18.32$ & $156.05 \pm 18.14$ & $339.65 \pm 32.84$ & $88.96 \pm 8.14$ & $5.50 \pm 2.45$ \\
\hline $\mathrm{CC}$ & $98.67 \pm 16.44^{*}$ & $133.33 \pm 10.15^{\star}$ & $294.57 \pm 31.24^{*}$ & $75.93 \pm 7.79^{*}$ & $3.42 \pm 1.54^{*}$ \\
\hline \multicolumn{6}{|l|}{ rs2236055 } \\
\hline AA & $120.04 \pm 19.66$ & $153.45 \pm 17.20$ & $331.19 \pm 28.64$ & $88.32 \pm 8.82$ & $4.97 \pm 2.35$ \\
\hline$A G$ & $120.11 \pm 20.26$ & $150.58 \pm 19.18$ & $330.88 \pm 34.66$ & $87.05 \pm 10.19$ & $4.91 \pm 2.50$ \\
\hline GG & $118.99 \pm 19.57$ & $149.42 \pm 20.05$ & $327.95 \pm 45.27$ & $87.16 \pm 10.21$ & $5.09 \pm 2.19$ \\
\hline
\end{tabular}

SNP: single nucleotide polymorphism; GPT: glutamic pyruvic transaminase; GOT: glutamic oxaloacetic transaminase; TB: total bilirubin; LA: lactic acid; the first genotype was considered as reference to conduct statistical analysis. Statistical analysis was carried out with one-way ANOVA ( $\left.{ }^{*} \mathrm{P}<0.05\right)$.

Table 4. Correlation analysis between haplotypes of MFN2 gene polymorphisms and the risk of acute liver failure.

\begin{tabular}{lccccc}
\hline Haplotype & Control group (freq) & Case group (freq) & P & OR & $95 \% \mathrm{Cl}$ \\
\hline CGACGGT & $2(0.013)$ & $5(0.036)$ & 0.148 & 2.393 & $0.710-8.068$ \\
CGGCGGC & $5(0.031)$ & $3(0.022)$ & 0.196 & $0 . .515$ & $0.186-1.429$ \\
CTACAAC & $4(0.025)$ & $5(0.036)$ & 0.436 & 1.492 & $0.543-4.101$ \\
CTACGGT & $6(0.038)$ & $3(0.022)$ & 0.069 & 0.402 & $0.147-1.104$ \\
CTGCAGC & $5(0.031)$ & $2(0.013)$ & 0.068 & 0.348 & $0.107-1.130$ \\
GTACAGC & $7(0.044)$ & $1(0.007)$ & $<0.001$ & 0.08 & $0.014-0.443$ \\
GTGTGGC & $2(0.013)$ & $10(0.072)$ & $<0.001$ & 5.644 & $1.846-17.258$ \\
\hline
\end{tabular}

freq: frequency; OR: odds ratio; $\mathrm{Cl}$ : confidence interval. The haplotypes from top to bottom correspond to rs 873457 , rs2336384, rs1474868, rs4846085, rs4240897, rs2236055, and rs873458, respectively.

Table 5. Binary logistic regression analysis of risk factors for acute liver failure.

\begin{tabular}{lllrrrrr}
\hline Factor & B & SE & Wald & Sig. & OR & \multicolumn{2}{c}{$95 \% \mathrm{Cl}$ for OR } \\
\cline { 4 - 8 } & & & & & & Lower & Upper \\
\hline Blood ammonia & 0.492 & 0.093 & 28.099 & $<0.001$ & 1.636 & 1.364 & 1.962 \\
LA & 2.36 & 0.347 & 46.233 & $<0.001$ & 10.591 & 5.364 & 20.912 \\
rs873457 & -0.982 & 0.269 & 13.354 & $<0.001$ & 0.375 & 0.221 & 0.634 \\
rs4846085 & -0.643 & 0.277 & 5.378 & 0.020 & 0.526 & 0.305 & 0.905 \\
\hline
\end{tabular}

LA: lactic acid; B: partial regression coefficient; SE: standard error; Sig: significance; OR: odds ratio; Cl: confidence interval. 

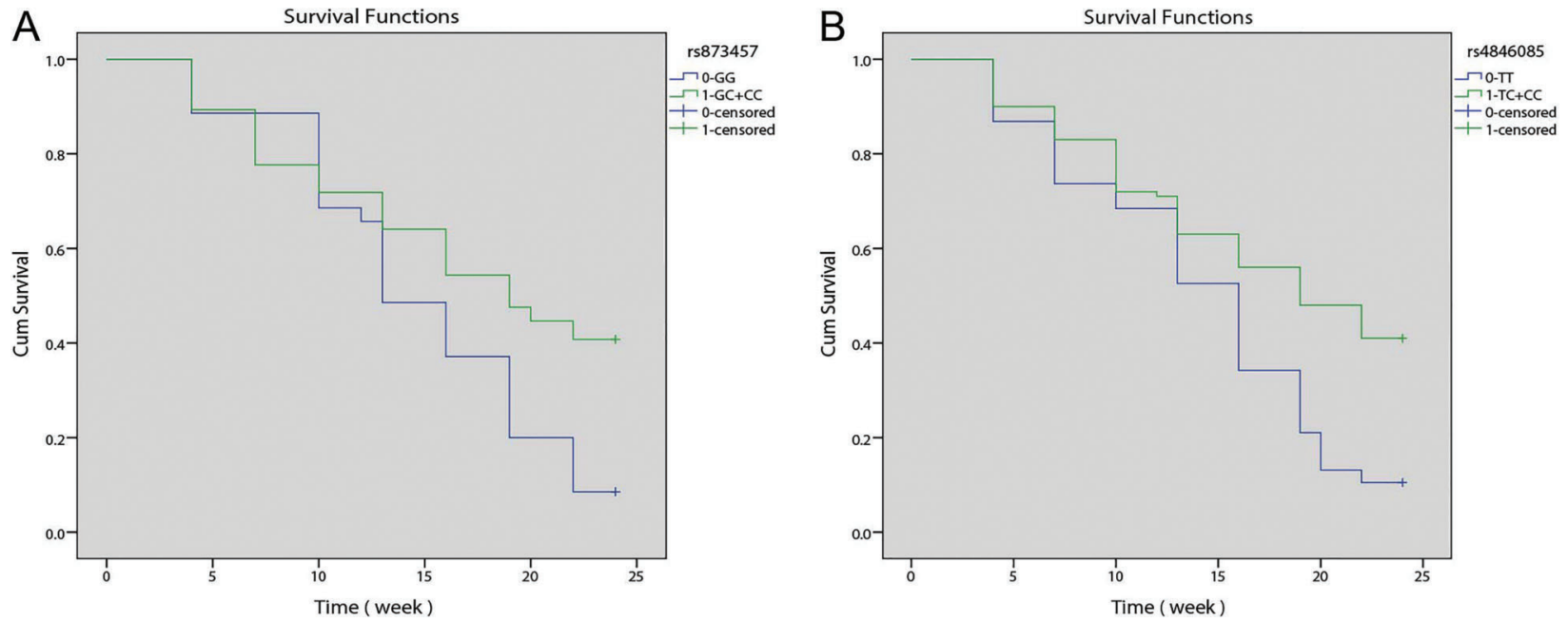

Figure 1. Kaplan-Meier curves of short-term prognosis of acute liver failure patients with different genotypes of mitofusin 2 (MFN2) polymorphisms. A, Comparison of survival rates in patients with the GG genotype and GC + CC genotypes of rs873457; $B$, comparison of survival rates in patients with the TT genotype and the TC + CC genotypes of rs 4846085 .

Table 6. Cox proportional hazards survival regression of short-term prognosis of acute liver failure.

\begin{tabular}{|c|c|c|c|c|c|c|c|}
\hline \multirow[t]{2}{*}{ Factor } & \multirow[t]{2}{*}{ B } & \multirow[t]{2}{*}{ SE } & \multirow[t]{2}{*}{ Wald } & \multirow[t]{2}{*}{ Sig. } & \multirow[t]{2}{*}{ OR } & \multicolumn{2}{|c|}{$95 \% \mathrm{Cl}$ for $\mathrm{OR}$} \\
\hline & & & & & & Lower & Upper \\
\hline GPT (U/L) & 0.005 & 0.005 & 0.701 & 0.402 & 1.005 & 0.994 & 1.015 \\
\hline GOT (U/L) & -0.001 & 0.006 & 0.045 & 0.831 & 0.999 & 0.987 & 1.01 \\
\hline $\mathrm{TB}(\mu \mathrm{mol} / \mathrm{L})$ & -0.004 & 0.004 & 1.509 & 0.219 & 0.996 & 0.989 & 1.003 \\
\hline Blood ammonia ( $\mu \mathrm{mol} / \mathrm{L})$ & 0.012 & 0.012 & 0.933 & 0.334 & 1.012 & 0.988 & 1.036 \\
\hline $\mathrm{LA}(\mu \mathrm{mol} / \mathrm{L})$ & 0.017 & 0.046 & 0.145 & 0.703 & 1.018 & 0.93 & 1.113 \\
\hline rs873457 & -0.669 & 0.234 & 8.174 & 0.004 & 0.512 & 0.324 & 0.81 \\
\hline rs4846085 & -0.663 & 0.233 & 8.084 & 0.004 & 0.515 & 0.326 & 0.814 \\
\hline
\end{tabular}

GPT: glutamic pyruvic transaminase; GOT: glutamic oxaloacetic transaminase; TB: total bilirubin; LA: lactic acid; B: partial regression coefficient; SE: standard error; Sig: significance; OR: odds ratio; $\mathrm{Cl}$ : confidence interval.

of ALF patients (all $\mathrm{P}>0.05$ ), but the rs4846085 and rs873457 polymorphisms were both independent factors affecting the prognosis of ALF patients (all $\mathrm{P}<0.05$ ).

\section{Discussion}

The findings of this study revealed that MFN2 gene polymorphisms (rs873457, rs2336384, rs1474868, rs4846085 and rs2236055) might be associated with ALF and that the rs873457 and rs4846085 polymorphisms are correlated with the risk and prognosis of ALF.

In this study, we selected seven SNPs of the MFN2 gene in ALF patients and analyzed the relationship between MFN2 SNP allele distribution, haplotype and ALF. The results indicated that rs873457, rs2336384, rs1474868, rs4846085 and rs2236055 were closely correlated with ALF. Current opinions suggest that if a disease-related mutation is located in the non-coding region, the mutation would function by regulating the transcription of the gene instead of affecting the translation of the gene (15). In our study, rs873457, rs2336384, rs1474868, rs4846085 and rs2236055 were all located in the transcriptional regulatory region of MFN2. To be more specific, they were located at the TATA box, the CCAAT box and the start of transcription at the second intron, which is in charge of regulating the transcription of MFN2. MFN2 gene transcription might be down-regulated when mutations occur at these SNPs, which might lead to the malfunction of mitochondria (16). Mitochondria are often called "the powerhouse of the cell" because they generate most of the cell's supply of adenosine triphosphate (ATP). The amount of ATP determines the responsiveness of the cell toward stimuli, and cells will undergo severe damage due to the lack of ATP. Currently, it is 
believed that the decreased level of MFN2 gene expression leads to the malfunction of mitochondria and increases in the ROS levels in the cell, which result in liver cell damage and ALF (17). Decreased expression of the MFN2 gene also leads to RAS-mediated PI3K/Akt activation, which results in abnormal cell cycle progression and malfunctions in multiple organs (18). Moreover, the concentration of $\mathrm{Ca}^{2+}$ is believed to play a critical role in oxidative metabolism, and when down-regulating the expression of the MFN2 gene, $\mathrm{Ca}^{2+}$ in the mitochondria accumulates, which leads to the elevation of oxidative stress and cell damage (19).

By comparing liver functions and different genotypes at rs873457 and rs4846085, the CC genotype was found to contribute to the decrease of liver function indexes, while patients with the GG genotype of rs873457 or the TT genotype of rs4846085 had higher coagulation function, GPT, GOT, TB, blood ammonia and LA levels. However, at rs2336384, rs1474868 and rs2236055, the variation of genotypes did not affect liver function significantly. We presume that the mechanism by which rs873457 or rs4846085 affects liver function may have to do with their location in the MFN2 gene intron, which is responsible for the transcriptional regulation of the MFN2 gene (11). The liver is the main organ in charge of metabolism in the human body; therefore, liver injury results in the elevation of coagulation function, GPT and GOT (20). Moreover, the liver is also responsible for urea and lactate metabolism, and a large area of damage in the liver also results in the elevation of blood ammonia and lactate levels (21). Therefore, multiple indexes could be used in the evaluation of

\section{References}

1. Mas A, Rodes J. Fulminant hepatic failure. Lancet 1997; 3499058: 1081-1085, doi: 10.1016/S0140-6736(96)08054-3.

2. Bernal W., Auzinger G, Dhawan A, Wendon J. Acute liver failure. Lancet 2010; 3769736: 190-201, doi: 10.1016/ S0140-6736(10)60274-7.

3. Lee WM, Squires RH Jr, Nyberg SL, Doo E, Hoofnagle JH. Acute liver failure: Summary of a workshop. Hepatology 2008; 474: 1401-1415, doi: 10.1002/hep.22177.

4. Ni HM, Williams JA, Yang H, Shi YH, Fan J, Ding WX. Targeting autophagy for the treatment of liver diseases. Pharmacol Res 2012; 66: 463-474, doi: 10.1016/j.phrs. 2012.07.003.

5. Santel A, Fuller MT. Control of mitochondrial morphology by a human mitofusin. J Cell Sci 2001; 114(Part 5): 867-874.

6. Auger C, Alhasawi A, Contavadoo M, Appanna VD. Dysfunctional mitochondrial bioenergetics and the pathogenesis of hepatic disorders. Front Cell Dev Biol 2015; 340, doi: 10.3389/fcell.2015.00040.

7. Liu X, Liu Z, Hou W, Wang K, Ding W, Chen D, et al. [Changes in mitochondria fusion protein-2 hepatic expression in conditions of liver cirrhosis and acute on chronic liver failure]. Zhonghua Gan Zang Bing Za Zhi 2014; 229: 671-675, doi: 10.3760/cma.j.issn.1007-3418.2014.09.008. liver function and ALF patient prognosis. These results were consistent with our analyses, which demonstrated that the rs873457 or rs4846085 CC genotypes are protective factors for ALF. Patients with the rs873457 GG genotype or the rs4846085 TT genotype have lower survival rates, indicating that the rs873457 or rs4846085 polymorphisms may be independent factors that correlate with ALF risk and prognosis.

The haplotype analysis demonstrated that GTACAGC was a protective factor for ALF, while GTGTGGC was a risk factor for ALF. Other haplotypes of the MFN2 gene and their relationships with ALF still remain unclear. Haplotype analysis has been valued as a good method in genetic studies of complex diseases with multiple variants, and this consensus may lead to the development of a more efficient strategy to identify genetic variants that increase the risk of human diseases (22). Moreover, Wang et al. (11) verified that MFN2 haplotypes were risk factors for hypertension, which suggests that SNP haplotypes of the MFN2 gene may also be correlated with other diseases.

ALF is a fatal disease that is associated with the rapid arrest of normal hepatic function, and is one of the most challenging emergencies encountered in clinical practice. Presently, effective prevention and therapy against ALF are not available but are urgently needed. The positive relationship between MFN2 gene polymorphisms and the occurrence of ALF will provide a new genetic tool to identify groups at high risk for ALF and shed light on the early prediction of ALF patients and the prevention of this disease.

8. Sebastian D, Hernandez-Alvarez MI, Segales J, Sorianello E, Munoz JP, Sala D, et al. Mitofusin 2 (Mfn2) links mitochondrial and endoplasmic reticulum function with insulin signaling and is essential for normal glucose homeostasis. Proc Natl Acad Sci U S A 2012; 10914: 5523-5528, doi: 10.1073/pnas.1108220109.

9. Li P, Zhu S, Wu X, Zhu X, Li J, Pan L, et al. Association of polymorphisms in mitofusin-2 gene with type 2 diabetes in Han Chinese. J Biomed Biotechnol 2012; 2012: 205752, doi: $10.1155 / 2012 / 205752$.

10. Li J, Ke W, Zhou Q, Wu Y, Luo H, Zhou H, et al. Tumour necrosis factor-alpha promotes liver ischaemia-reperfusion injury through the PGC-1alpha/Mfn2 pathway. J Cell Mol Med 2014; 18: 1863-1873, doi: 10.1111/jcmm.12320.

11. Wang Z, Liu Y, Liu J, Liu K, Wen J, Wen S, et al. HSG/Mfn2 gene polymorphism and essential hypertension: a casecontrol association study in Chinese. $J$ Atheroscler Thromb 2011; 181: 24-31, doi: 10.5551/jat.5611.

12. Polke JM, Laura M, Pareyson D, Taroni F, Milani M, Bergamin G, et al. Recessive axonal Charcot-Marie-Tooth disease due to compound heterozygous mitofusin 2 mutations. Neurology 2011; 77: 168-173, doi: 10.1212/WNL. 0b013e3182242d4d. 
13. Singh T, Gupta N, Alkhouri N, Carey WD, Hanouneh IA. A guide to managing acute liver failure. Cleve Clin J Med 2016; 83: 453-462, doi: 10.3949/ccjm.83a.15101.

14. Polson J, Lee WM. American Association for the Study of Liver. AASLD position paper: the management of acute liver failure. Hepatology 2005; 41: 1179-1197, doi: 10.1002/hep. 20703.

15. Drogemuller C, Reichart U, Seuberlich T, Oevermann A, Baumgartner $\mathrm{M}$, Kuhni Boghenbor $\mathrm{K}$, et al. An unusual splice defect in the mitofusin 2 gene (MFN2) is associated with degenerative axonopathy in Tyrolean Grey cattle. PLoS One 2011; 64: e18931, doi: 10.1371/journal.pone.0018931.

16. Pang W, Zhang Y, Zhao N, Darwiche SS, Fu X, Xiang W. Low expression of Mfn2 is associated with mitochondrial damage and apoptosis in the placental villi of early unexplained miscarriage. Placenta 2013; 347: 613-618, doi: 10.1016/j.placenta.2013.03.013.

17. Zhang $Y$, Jiang L, Hu W, Zheng Q, Xiang W. Mitochondrial dysfunction during in vitro hepatocyte steatosis is reversed by omega-3 fatty acid-induced up-regulation of mitofusin 2 .
Metabolism 2011; 606: 767-775, doi: 10.1016/j.metabol. 2010.07.026.

18. de Brito OM, Scorrano L. Mitofusin-2 regulates mitochondrial and endoplasmic reticulum morphology and tethering: the role of Ras. Mitochondrion 2009; 93: 222-226, doi: 10.1016/j.mito.2009.02.005.

19. Rizzuto R, De Stefani D, Raffaello A, Mammucari C. Mitochondria as sensors and regulators of calcium signalling. Nat Rev Mol Cell Biol 2012; 139: 566-578, doi: 10.1038/nrm3412.

20. Olson JC, Kamath PS. Acute-on-chronic liver failure: concept, natural history, and prognosis. Curr Opin Crit Care 2011; 172: 165-169, doi: 10.1097/MCC.0b013e328344b42d.

21. Bernal W, Donaldson N, Wyncoll D, Wendon J. Blood lactate as an early predictor of outcome in paracetamol-induced acute liver failure: a cohort study. Lancet 2002; 3599306: 558-563, doi: 10.1016/S0140-6736(02)07743-7.

22. Zhao $\mathrm{H}$, Pfeiffer $\mathrm{R}$, Gail $\mathrm{MH}$. Haplotype analysis in population genetics and association studies. Pharmacogenomics 2003; 4: 171-178, doi: 10.1517/phgs.4.2.171.22636. 